\title{
Finite element approach to Bragg edge neutron strain tomography
}

\author{
$\begin{array}{lll}\text { R. Aggarwal } & \text { M. H. Meylan } & \text { C. M. Wensrich } \\ & \end{array}$ \\ B. P. Lamichhane ${ }^{4}$
}

(Received 27 February 2019; revised 9 May 2020)

\begin{abstract}
A number of techniques and applications in neutron imaging that exploit wavelength resolved measurements have been developed recently. One such technique, known as energy resolved neutron imaging, receives ample attention because of its capability to not only visualise but to also quantify physical attributes with spatial resolution. The objective of this article is to develop a reconstruction algorithm for elastic strain tomography from Bragg edge neutron transmission strain images obtained from a pulsed neutron beam with high resolution. This technique has several advantages over those using monochromatic neutron beams from continuous sources; for example, finer wavelength resolution. In contrast to the conventional radon based computed
\end{abstract}

DOI:10.21914/anziamj.v60i0.14054, C Austral. Mathematical Soc. 2020. Published 2020-06-17, as part of the Proceedings of the 18th Biennial Computational Techniques and Applications Conference. ISSN 1445-8810. (Print two pages per sheet of paper.) Copies of this article must not be made otherwise available on the internet; instead link directly to the DOI for this article. 
tomography, wherein neutron transmission revolves around the inversion of the longitudinal ray transform that has uniqueness issues, the reconstruction in the proposed algorithm is based on the least squares approach, constrained by an equilibrium formulated through the finite element method.

\section{Contents}

1 Introduction

C280

2 Strain reconstruction algorithm

C281

2.1 Strain measurement. . . . . . . . . . . . . .

3 Model

C282

3.1 Simulation measurement . . . . . . . . . . . . . C282

3.2 Cantilevered beam problem . . . . . . . . . . C284

3.3 Constructing a system of equations . . . . . . . . C285

4 Simulation and analysis

C286

5 Conclusion

C288

\section{Introduction}

Energy resolved transmission imaging using time-of-flight (TOF) spectroscopy of pulsed neutrons can give high wavelength-resolution Bragg edge transmission spectra of polycrystalline materials. In these experiments, the term Bragg edge refers to a sudden increase in the relative transmission of neutron beams passing through polycrystalline solids as a function of wavelength. These edges are formed through diffraction where a neutron of wavelength $\lambda$ is coherently scattered by crystal planes with lattice spacing $d_{k h l}$, provided that the scattering angle $\theta$ satisfies Bragg's law $\lambda=2 \mathrm{~d}_{\mathrm{khl}} \sin \theta$. A sudden increase in transmission occurs once $\lambda>2 d_{\text {khl }}$ as a neutron cannot be scattered by 
more than $180^{\circ}$ [8]. Tomographic reconstruction determines a map of an unknown quantity within an object from lower dimensional projections. A well known example is computed tomography, where a set of flat two dimensional $\mathrm{X}$-ray images are analysed to build a three dimensional image of the scalar density.

Strain imaging raises the prospect of strain tomography, and over the past decade several attempts have been made to solve the resulting tensor reconstruction problem. Lionheart and Withers [5] demonstrated that it is not possible to reconstruct strain in the general setting since the strain field produced by any given set of projections is not unique. Hence, additional information (equilibrium [2] or compatibility constraints) is required to reconstruct the required strain field from all the possibilities. Abbey et al. [1] developed an algorithm using different basis functions along with compatibility constraints [3]. Unknown strain can also be reconstructed by using a machine learning technique involving Gaussian processes [4, 6].

In this article, we describe a method to tomographically reconstruct the elastic strain from a series of Bragg edge strain measurements by using finite element discretisation. The proposed algorithm is tested on cantilevered beam simulated data in two dimensions and is shown to be capable of reconstructing a strain tensor field after imposing equilibrium conditions.

\section{Strain reconstruction algorithm}

\subsection{Strain measurement}

The strain is measured in the direction of the scattering vector, which bisects the angle between the incident and diffracted beams. Lattice spacing $d$ is determined from the measured angular position of the Bragg reflection by illuminating the material with a monochromatic collimated beam of neutrons [7]. The strain free lattice spacing is $d_{0}$. In a stressed material, lattice spacings are altered and a shift in each Bragg peak position occurs. 
Hence, for TOF instruments the elastic strain is

$$
\epsilon=\frac{\mathrm{d}-\mathrm{d}_{0}}{\mathrm{~d}_{0}}=\frac{\triangle \mathrm{d}}{\mathrm{d}_{0}} .
$$

At a fixed scattering angle $\theta$, the TOF $t$ is proportional to the lattice spacing $d$; that is,

$$
\mathrm{t} \propto \mathrm{d} \sin \theta
$$

The average strain within a body, as measured by Bragg edge neutron transmission, is idealised as a line integral typically known as a longitudinal ray transform (LRT). This line integral captures the component of strain along the line $s$ in the direction of unit normal $\hat{n}=\left(n_{1}, n_{2}\right)$. For a ray in the direction $\hat{\boldsymbol{n}}$ which enters the sample at $\boldsymbol{x}_{\mathbf{a}}$ and has length $\mathrm{L}$ inside the sample, as shown in Figure 1, the LRT is

$$
\Gamma_{\epsilon}=\frac{1}{\mathrm{~L}} \int_{0}^{\mathrm{L}} \hat{\mathrm{n}} \epsilon\left(\boldsymbol{x}_{\mathrm{a}}+s \hat{\mathrm{n}}\right) \hat{\mathrm{n}}^{\mathrm{T}} \mathrm{ds},
$$

where

$$
\epsilon(x, y)=\left[\begin{array}{ll}
\epsilon_{11}(x, y) & \epsilon_{12}(x, y) \\
\epsilon_{21}(x, y) & \epsilon_{22}(x, y)
\end{array}\right] \in \mathbb{R}^{2 \times 2}
$$

is the symmetric strain tensor field with $\epsilon_{12}(x, y)=\epsilon_{21}(x, y)$. The finite element rectangular mesh plate used for the algorithm is shown in Figure 1.

\section{Model}

\subsection{Simulation measurement}

For reconstruction, we assume that the strain is expressed as a linear combination of basis functions, where $(x, y)$ is an arbitrary point in the sample geometry displayed in Figure 1 and where $\boldsymbol{x}_{\mathrm{a}}=\left(\boldsymbol{x}_{\mathrm{a}}, \boldsymbol{y}_{\mathrm{a}}\right)$ and $\boldsymbol{x}_{\mathrm{b}}=\left(\boldsymbol{x}_{\mathrm{b}}, \boldsymbol{y}_{\mathrm{b}}\right)$ are entry and exit points of the ray, respectively, with $\hat{\boldsymbol{n}}=\left(\boldsymbol{x}_{\mathrm{b}}-\boldsymbol{x}_{\mathrm{a}}\right) /\left\|\boldsymbol{x}_{\mathrm{b}}-\boldsymbol{x}_{\mathrm{a}}\right\|=$ $\left(n_{1}, n_{2}\right)$. Define the strain field components as

$$
\epsilon_{11}(x, y)=\beta_{1}+\beta_{2} x+\beta_{3} y+\beta_{4} x y,
$$




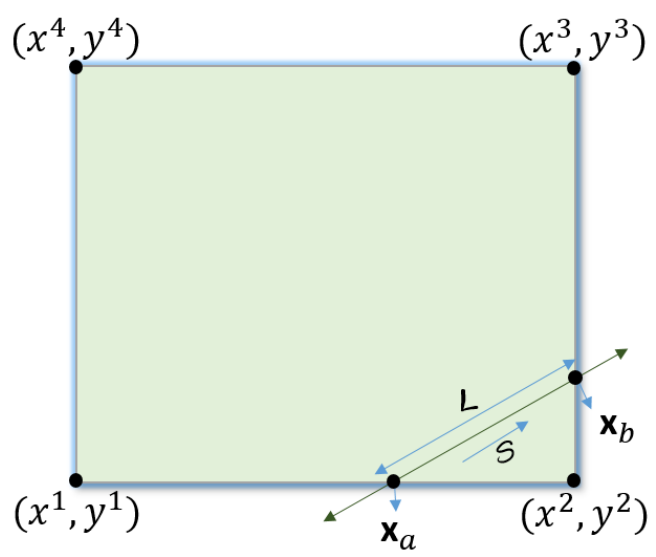

Figure 1: Longitudinal ray transform: (left) finite element mesh plate; and (right) sample geometry.

$$
\begin{aligned}
& \epsilon_{12}(x, y)=\gamma_{1}+\gamma_{2} x+\gamma_{3} y+\gamma_{4} x y=\epsilon_{21}(x, y), \\
& \epsilon_{22}(x, y)=\eta_{1}+\eta_{2} x+\eta_{3} y+\eta_{4} x y,
\end{aligned}
$$

where $\left\{\beta_{i}, \gamma_{i}, \eta_{i}\right\}, i=\{1,2,3,4\}$, are unknowns which needs to be determined. The main problem is to find the strain components in the line integral (3). Expanding the terms in this line integral gives

$$
\Gamma_{\epsilon}=\frac{1}{L} \int_{0}^{L}\left(\sum_{i, j=1}^{2} n_{i} \epsilon_{i j}\left(x_{a}+s \hat{n}\right) n_{j}\right) d s .
$$

The strain components defined in equation (4) are now rewritten in matrix form. Let $\left(x^{r}, y^{r}\right), r \in\{1,2,3,4\}$, be the node values at the four corners of the rectangle in the sample geometry (Figure 1), and $\epsilon_{k \ell}^{r}=\epsilon_{k \ell}\left(x^{r}, y^{r}\right)$ for $r \in\{1,2,3,4\}, k, \ell \in\{1,2\}$, represents the value of the known strain at the 
rth node position. Hence, matrices of strain components are

$$
\begin{aligned}
& {\left[\begin{array}{l}
\epsilon_{11}^{1} \\
\epsilon_{11}^{2} \\
\epsilon_{11}^{3} \\
\epsilon_{11}^{4}
\end{array}\right]=\left[\begin{array}{llll}
1 & x^{1} & y^{1} & x^{1} y^{1} \\
1 & x^{2} & y^{2} & x^{2} y^{2} \\
1 & x^{3} & y^{3} & x^{3} y^{3} \\
1 & x^{4} & y^{4} & x^{4} y^{4}
\end{array}\right]\left[\begin{array}{l}
\beta_{1} \\
\beta_{2} \\
\beta_{3} \\
\beta_{4}
\end{array}\right], \quad\left[\begin{array}{l}
\epsilon_{12}^{1} \\
\epsilon_{12}^{2} \\
\epsilon_{12}^{3} \\
\epsilon_{12}^{4}
\end{array}\right]=\left[\begin{array}{llll}
1 & x^{1} & y^{1} & x^{1} y^{1} \\
1 & x^{2} & y^{2} & x^{2} y^{2} \\
1 & x^{3} & y^{3} & x^{3} y^{3} \\
1 & x^{4} & y^{4} & x^{4} y^{4}
\end{array}\right]\left[\begin{array}{l}
\gamma_{1} \\
\epsilon_{22} \\
\epsilon_{22}^{2} \\
\epsilon_{22}^{4}
\end{array}\right]=\left[\begin{array}{llll}
1 & x^{1} & y^{1} & x^{1} y^{1} \\
1 & x^{2} & y^{2} & x^{2} y^{2} \\
1 & x^{3} & y^{3} & x^{3} y^{3} \\
1 & x^{4} & y^{4} & x^{4} y^{4}
\end{array}\right],\left[\begin{array}{l}
\eta_{1} \\
\gamma_{2} \\
\gamma_{4}
\end{array}\right] .}
\end{aligned}
$$

The above matrices are rewritten as

$$
\begin{aligned}
& \tilde{\epsilon}_{11}=\mathbf{A} \tilde{\beta}, \quad \tilde{\epsilon}_{12}=\mathbf{A} \tilde{\gamma}, \quad \tilde{\epsilon}_{22}=\mathbf{A} \tilde{\eta}, \\
& \boldsymbol{A}^{-1} \tilde{\epsilon}_{11}=\tilde{\beta}, \quad \boldsymbol{A}^{-1} \tilde{\epsilon}_{12}=\tilde{\gamma}, \quad \boldsymbol{A}^{-1} \tilde{\epsilon}_{22}=\tilde{\eta},
\end{aligned}
$$

where all unknowns $\{\tilde{\beta}, \tilde{\gamma}, \tilde{\eta}\}$ are written in terms of known strain values at the node values $\left(x^{r}, y^{r}\right)$ and substituted into the exact integral expression (5).

\subsection{Cantilevered beam problem}

In this section we describe the Bragg edge measurements for the cantilevered beam problem. Geometrically, the line integral (5) resembles the area of a plane perpendicular to the $x y$ plane and extending from the ray path to the surface to the sample. Figure 2 shows a visualisation of a cantilevered beam of width $W$, thickness $t$, ray propagation distance co-ordinate $s$, ray distance inside beam $\mathrm{L}$, load $\mathrm{P}$ and a rectangular discretisation.

The Bragg edge measurements are approximated by

$$
\Gamma_{\epsilon} \approx \frac{1}{\mathrm{~L}} \sum_{j \in \mathrm{B}} \int_{0}^{\mathrm{L}_{j}} \hat{n}^{\top} \epsilon_{j} \hat{\mathrm{n}} \mathrm{d} s,
$$

where $B=\left\{B_{1}, B_{2}, \ldots, B_{N}\right\}$ is the set of all rectangular elements intersected by different rays with associated length $L_{j}$ in element $B_{j}$, as shown in Figure 2. 

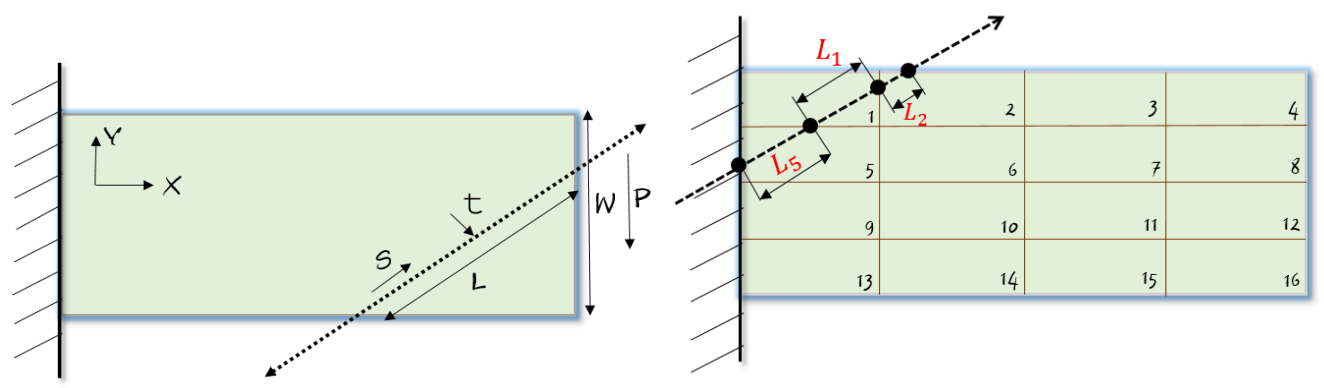

Figure 2: Cantilevered beam: (left) beam setup; and (right) rectangular beam elements $B_{j}$ are intersected by different neutron rays with associated lengths $L_{j}$, with this example showing a ray intersecting the $j=1,2,5$ elements.

Hence, for one ray which intersects $B_{j}$ with $j \in\{1,2,5\}$, as in the example shown in Figure 2, the Bragg edge measurement is approximated by

$$
\Gamma_{\epsilon} \approx \frac{1}{\mathrm{~L}}\left[\int_{0}^{\mathrm{L}_{1}} \hat{\mathrm{n}}^{\top} \epsilon_{1} \hat{\mathrm{n}} \mathrm{ds}+\int_{0}^{\mathrm{L}_{2}} \hat{\mathrm{n}}^{\top} \epsilon_{2} \hat{\mathrm{n}} \mathrm{ds}+\int_{0}^{\mathrm{L}_{5}} \widehat{n}^{\top} \epsilon_{5} \hat{\mathrm{n}} \mathrm{ds}\right],
$$

where $L=\sum_{j} L_{j}$. Using the exact integral expression and evaluation of basis functions for each element, the integral is reformulated in terms of $\beta^{j}$, $\gamma^{j}$ and $\eta^{j}$ which depend on the node values and the simple strain evaluation at the particular node point.

\subsection{Constructing a system of equations}

For $\mathrm{N}$ rays we construct an $\mathrm{N}$ dimensional line integral vector with each element corresponding to one ray

$$
\left[\begin{array}{c}
\Gamma_{1} \\
\vdots \\
\Gamma_{\mathrm{N}}
\end{array}\right]=\left[\begin{array}{ccc}
\mathrm{C} \beta_{1}^{1} & \cdots & \mathrm{C} \eta_{4}^{1} \\
\vdots & \ddots & \vdots \\
\mathrm{C} \beta_{1}^{\mathrm{N}} & \cdots & \mathrm{C} \eta_{4}^{\mathrm{N}}
\end{array}\right]\left[\begin{array}{c}
\beta_{1}^{1} \\
\vdots \\
\eta_{4}^{\mathrm{N}}
\end{array}\right],
$$

where, for $i \in\{1,2,3,4\}$ and $j \in\{1,2, \ldots, N\}, C \beta_{i}^{j}$ represents the coefficients of $\beta_{i}^{j}$ and $C \gamma_{i}^{j}$ represents the coefficients of $\gamma_{i}^{j}$. We rewrite the above equation 
as

$$
\hat{\Gamma}=\mathbf{K} \phi,
$$

where $\hat{\Gamma}$ is a vector containing all of the Bragg edge strain measurements, $\mathbf{K}$ is a sparse coefficient matrix with elements that contain unit direction vector components and basis function evaluations, and $\phi$ is a vector containing all the unknowns for each element. Once the matrix $\mathbf{K}$ and vector $\hat{\Gamma}$ are formed, the problem is reduced to one of solving the linear algebraic system of equations for the unknown coefficients represented by vector $\phi$. In practice the system is usually overdetermined since the number of unknown coefficients is relatively small compared to the amount of experimental data available (i.e., $\mathbf{K}$ matrix will be sparse with only 12 non-zero values in each row corresponding to the coefficients of all unknowns in the integral).

Solutions $\phi$ were found by least-squares fitting where the problem is reduced to finding a vector $\phi^{*}$ such that

$$
\left\|\mathbf{K} \phi^{*}-\hat{\Gamma}\right\|_{2}=\min \|\mathbf{K} \phi-\hat{\Gamma}\|_{2},
$$

where the constraints to the optimization problem (7) are obtained by imposing the equilibrium equations

$$
\frac{\partial}{\partial x}\left(\epsilon_{x x}+v \epsilon_{y y}\right)+\frac{\partial}{\partial y}(1-v) \epsilon_{x y}=0, \quad \frac{\partial}{\partial y}\left(\epsilon_{y y}+v \epsilon_{x x}\right)+\frac{\partial}{\partial x}(1-v) \epsilon_{x y}=0 .
$$

To reconstruct the strain we further integrate these equilibrium equations over each element $B$, which leads to the weak formulation

$$
\begin{aligned}
& \iint_{B}\left[\beta_{2}+\beta_{4} y+v\left(\eta_{2}+\eta_{4} y\right)+(1-v)\left(\gamma_{3}+\gamma_{4} x\right)\right] d x d y=0 \\
& \iint_{B}\left[\eta_{3}+\eta_{4} x+v\left(\beta_{3}+\beta_{4} x\right)+(1-v)\left(\gamma_{2}+\gamma_{4} y\right)\right] d x d y=0 .
\end{aligned}
$$

\section{Simulation and analysis}

To demonstrate the performance of the proposed algorithm, a well known 2D cantilevered beam problem is studied. We consider the $2 \mathrm{D}$ strain field for 

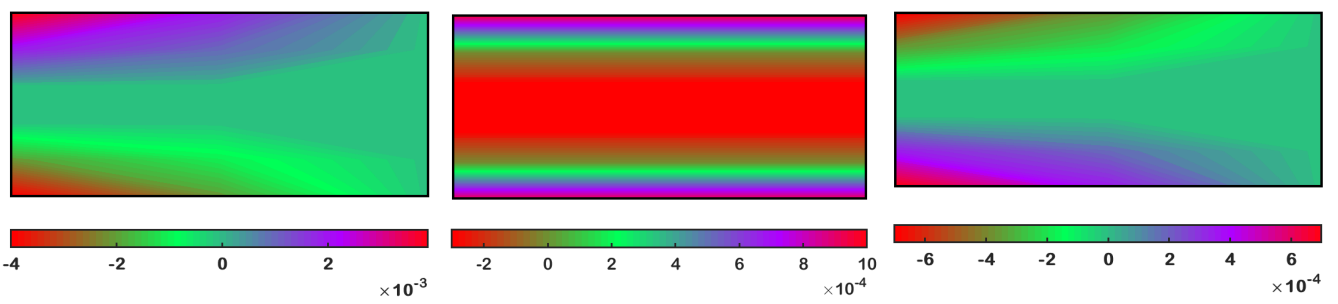

Figure 3: True strain fields (left) $\epsilon_{x x}$, (middle) $\epsilon_{x y}$ and (right) $\epsilon_{y y}$.
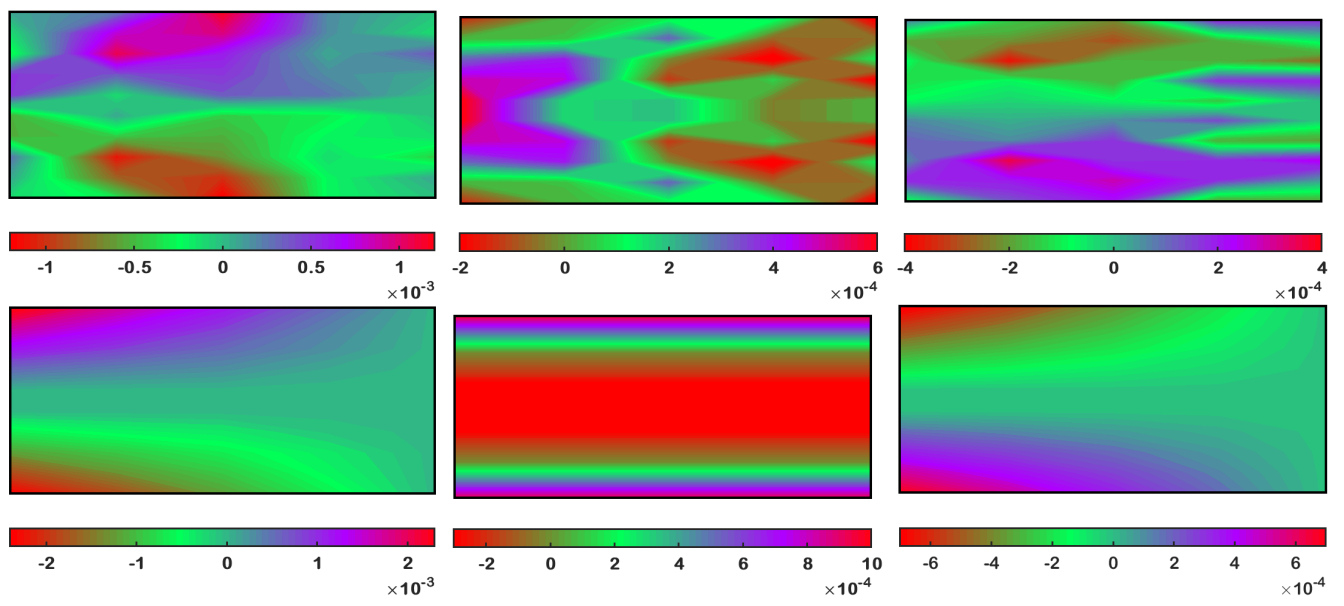

Figure 4: Reconstructed strain fields (left) $\epsilon_{x x}$, (middle) $\epsilon_{x y}$ and (right) $\epsilon_{y y}$ over a $4 \times 4$ rectangular mesh with Young's modulus E $=200 \mathrm{GPa}$, Poisson's ratio $v=0.3$ and with (top row) 20 projections and (bottom row) 60 projections.

rectangular beam geometry $[0,12] \times[0,10]$ with load $\mathrm{P}=2 \mathrm{kN}$, and thickness $\mathrm{t}=3 \mathrm{~mm}$ (Figure 2). This beam problem is excellent for testing the algorithm since the analytical solutions to the strain field exist and are shown in Figure 3. A finite element model of the system was constructed using the rectangular mesh. Reconstructed solutions to the strain fields for the cantilevered beam are shown in the Figure 4 for 20 and 60 projections. 
Average errors are shown in the bottom plots of Figures 5-8, with associated projections over $360^{\circ}$ shown in the top plots. A few different projection configurations are considered. For example, the projections shown in Figures 5 and 7 are based on constant angular increments, where every new angle divides one of the largest angular gaps according to the golden ratio. This guarantees that the maximum amount of new information is gained. The numerical errors are calculated using the root-mean-square error

$$
\text { Error }=\sqrt{\frac{\sum_{i=1}^{N}\left(T_{i}-R_{i}\right)^{2}}{N}},
$$

where $\mathrm{N}$ is the total number of nodes, $T_{i}$ is the true strain value and $R_{i}$ is the reconstructed strain value at the particular node $i$.

\section{Conclusion}

Features of a beam are revealed or enhanced in neutron transmission imaging by the wavelength resolved cross section. Numerical solutions were obtained from a finite element model with a structured rectangular mesh. While problem discretisation and numerical errors can undoubtedly contribute to an imperfect reconstruction (with more noise), additional error was caused mainly by a systematic error in the measurement environment. After some threshold, increasing the number of measurements (i.e., projections) nets little information about the sample. Rapid convergence to the true solution was observed as the number of projections were increased from the minimum. In summary, it was found that the reconstruction algorithm was extremely effective at achieving strain field reconstruction.

Acknowledgements The Australian Research Council supports this work through a Discovery Project Grant. RA's scholarship is supported by ARC grant, UNIPRS, UNSR5050 Central School. 

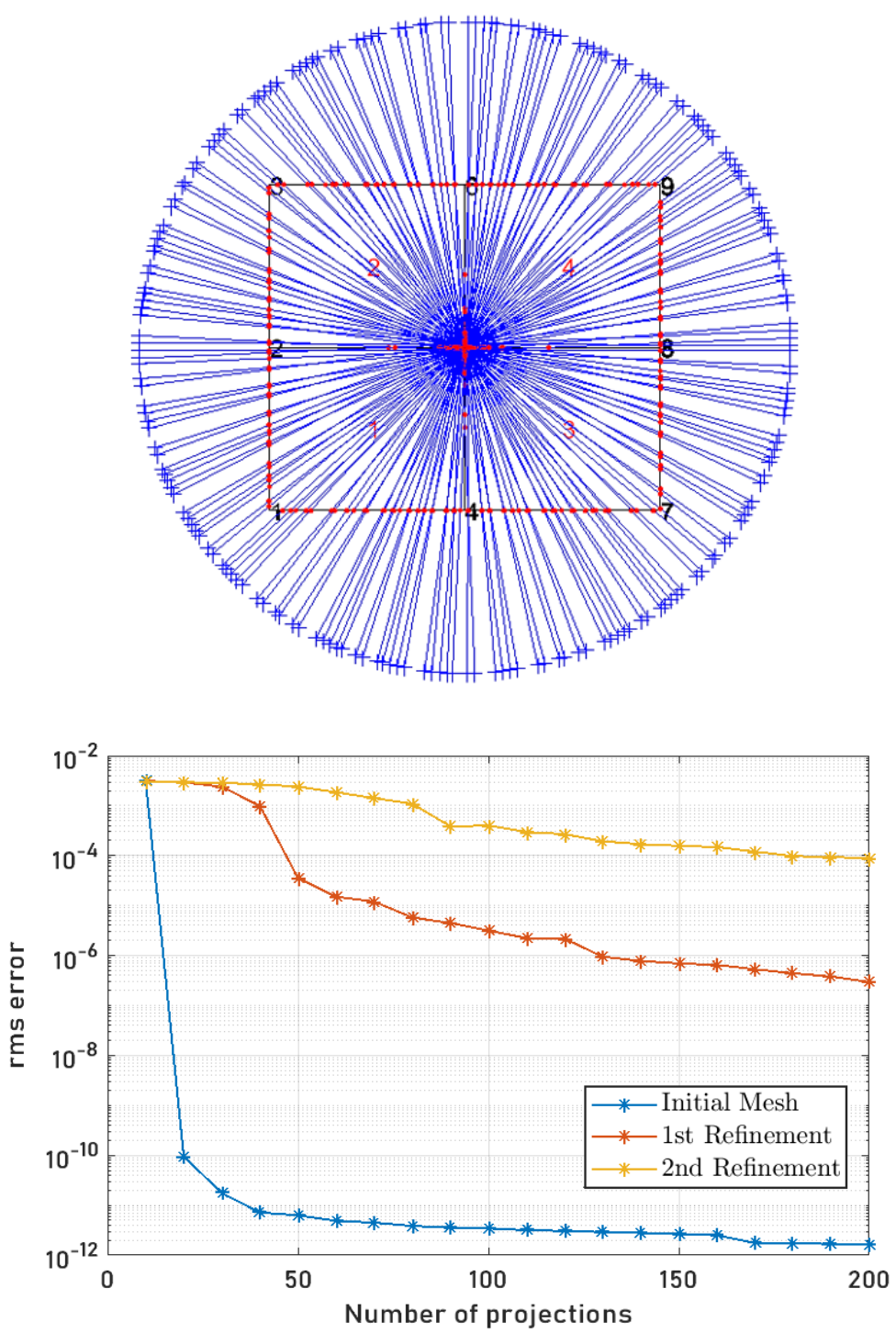

Figure 5: The top plot shows rays with projection angles based on the golden angle increment over $360^{\circ}$ and the bottom plot shows the convergence rate of the number of rays with respect to the mesh discretisation. 

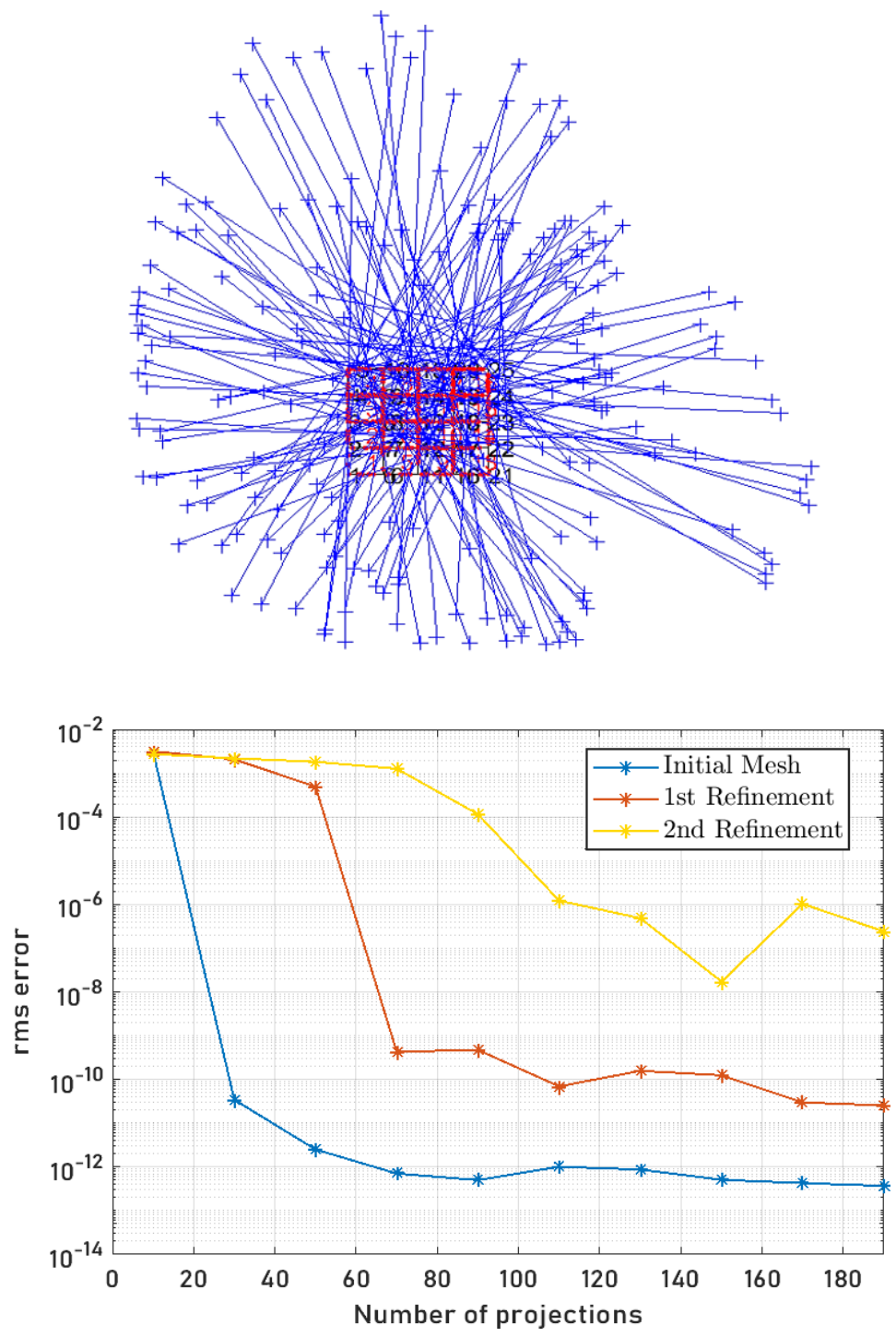

Figure 6: The top plot shows rays with random projections and the bottom plot shows the convergence rate of the number of projections with respect to the mesh discretisation. 

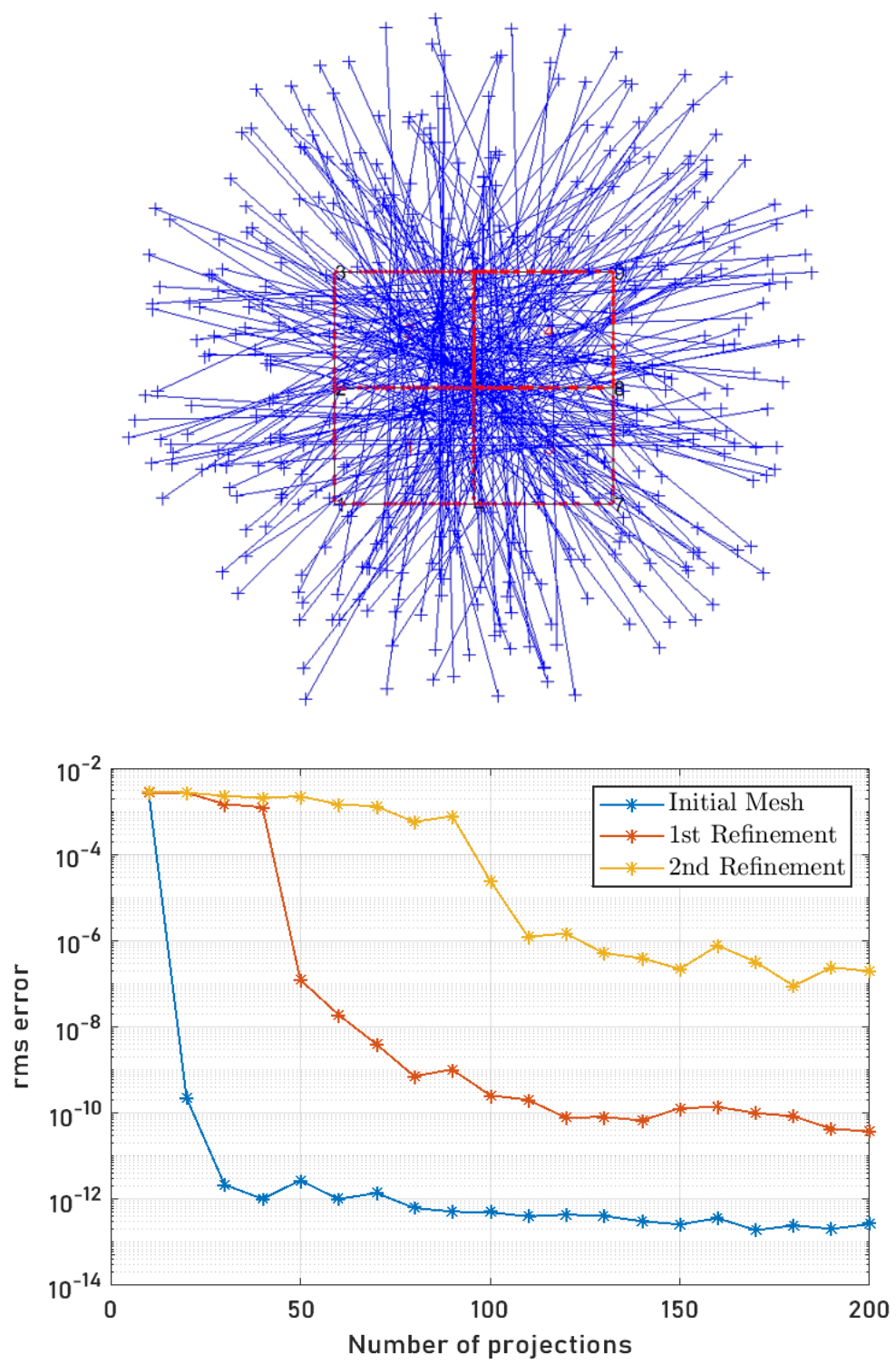

Figure 7: The top plot shows rays with projection angles based on the golden angle increment over $360^{\circ}$ and the bottom plot shows the convergence rate of the number of rays with respect to the mesh discretisation. 

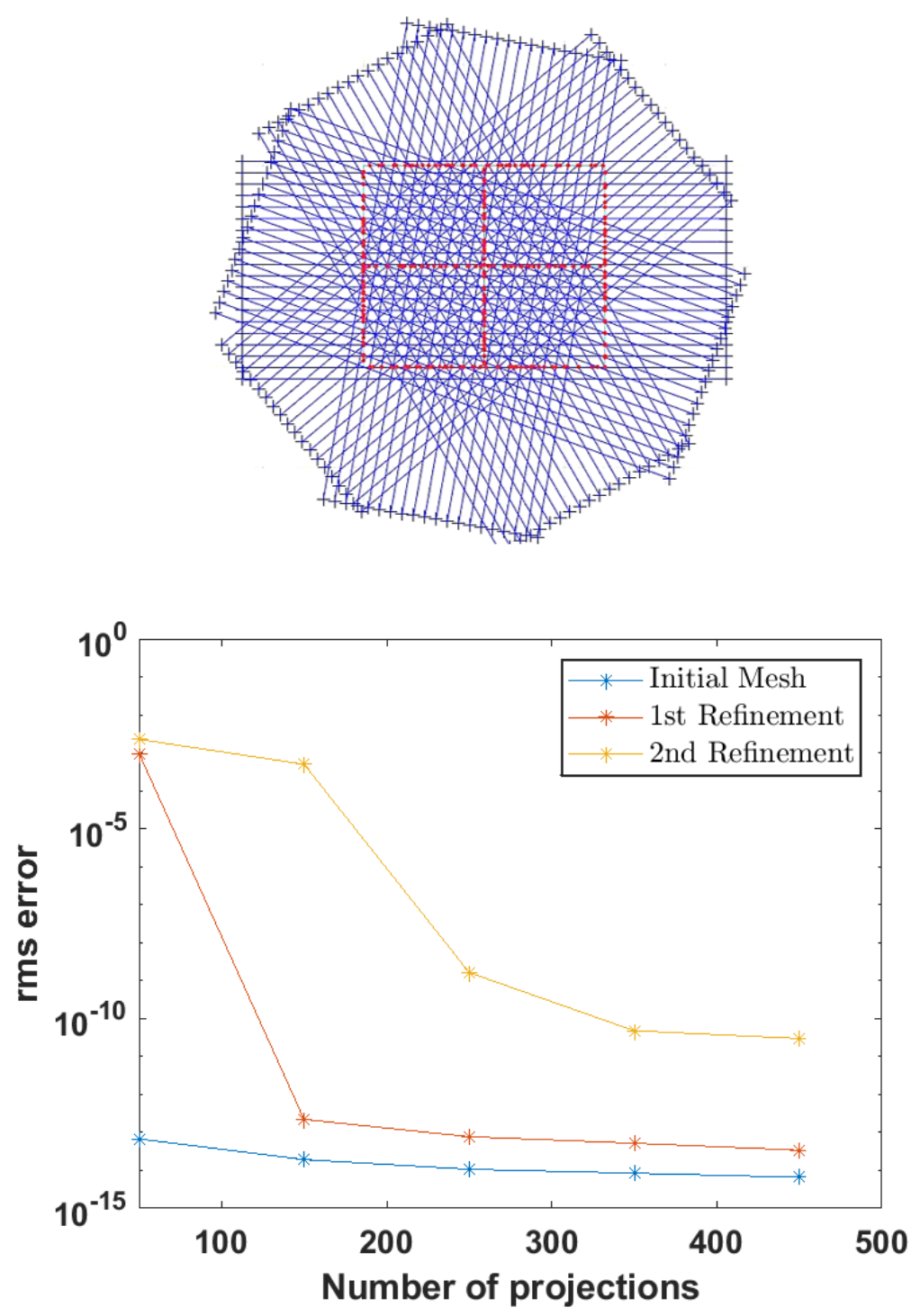

Figure 8: The top plot shows rays with different rotations of projected angle and the bottom plot shows the convergence rate of the number of projections with respect to the mesh discretisation. 


\section{References}

[1] B. Abbey, S. Y. Zhang, W. J. J. Vorster, and A. M. Korsunsky. "Feasibility study of neutron strain tomography". In: Proc. Eng. 1.1 (2009), pp. 185-188. DOI: 10.1016/j.proeng. 2009.06.043 (cit. on p. C281).

[2] R. Aggarwal, M. H. Meylan, B. P. Lamichhane, and C. M. Wensrich. "Energy resolved neutron imaging for strain reconstruction using the finite element method". In: J. Imag. 6.3 (2020), p. 13. DOI: 10.3390/jimaging6030013 (cit. on p. C281).

[3] J. N. Hendriks, A. W. T. Gregg, C. M. Wensrich, A. S. Tremsin, T. Shinohara, M. Meylan, E. H. Kisi, V. Luzin, and O. Kirsten. "Bragg-edge elastic strain tomography for in situ systems from energy-resolved neutron transmission imaging". In: Phys. Rev. Mat. 1 (2017), p. 053802. DOI: 10.1103/PhysRevMaterials.1.053802 (cit. on p. C281).

[4] C. Jidling, J. Hendriks, N. Wahlström, A. Gregg, T. B. Schön, C. Wensrich, and A. Wills. "Probabilistic modelling and reconstruction of strain". In: Nuc. Inst. Meth. Phys. Res. B (2018), pp. 141-155. DOI: 10.1016/j.nimb.2018.08.051 (cit. on p. C281).

[5] W. R. B. Lionheart and P. J. Withers. "Diffraction tomography of strain". In: Inv. Prob. 31 (2015), p. 045005. DOI: 10.1088/0266-5611/31/4/045005 (cit. on p. C281).

[6] C. E. Rasmussen and C. K. I. Williams. Gaussian processes for machine learning. MIT Press, 2006. URL:

https://mitpress.mit.edu/books/gaussian-processes-machinelearning (cit. on p. C281).

[7] C. M. Wensrich, E. Kisi, V. Luzin, and O. Kirstein. "Non-contact measurement of the stress within granular materials via neutron diffraction". In: AIP Conference Proceedings 1542 (2013), pp. 441-444. DOI: $10.1063 / 1.4811962$ (cit. on p. C281). 
[8] R. Woracek, J. Santisteban, A. Fedrigo, and M. Strobl. "Diffraction in neutron imaging - A review". In: Nuc. Inst. Meth. Phys. Res. A 878 (2018), pp. 141-158. DOI: 10.1016/j.nima.2017.07.040 (cit. on p. C281).

\section{Author addresses}

1. R. Aggarwal, School of Mathematics and Physical Sciences, The University of Newcastle, Australia. mailto:riya.aggarwal@uon.edu.au orcid:0000-0003-4261-8223

2. M. H. Meylan, School of Mathematics and Physical Sciences, The University of Newcastle, Australia. mailto:mike.meylan@newcastle.edu.au

3. C. M. Wensrich, School of Engineering, The University of Newcastle, Australia.

mailto: christopher. wensrich@newcastle.edu.au

4. B. P. Lamichhane, School of Mathematics and Physical Sciences, The University of Newcastle, Australia. mailto:bishnu.lamichhane@newcastle.edu.au 